\title{
Disability transitions in the oldest old in the general population. The Leiden 85-plus study
}

\author{
Anne H. van Houwelingen • Ian D. Cameron - Jacobijn Gussekloo • \\ Hein Putter • Susan Kurrle • Anton J. M. de Craen • Andrea B. Maier • \\ Wendy P. J. den Elzen • Jeanet W. Blom
}

Received: 15 January 2013 / Accepted: 22 July 2013 / Published online: 30 August 2013

(C) American Aging Association 2013. This article is published with open access at Springerlink.com

\begin{abstract}
Transitions between disability states in older people occur frequently. This study investigated predictors of disability transitions in the oldest old and was performed in the Leiden 85-plus study, a populationbased prospective cohort study among 597 participants aged 85 years. At baseline (age 85 years), data on sociodemographic characteristics and chronic diseases were obtained. Disabilities in basic activities of daily living (BADL) and instrumental activities of daily living (IADL) were measured annually for 5 years with the Groningen Activities Restriction Scale (GARS). Mortality data were obtained. A statistical multi-state model was used to assess the risks of transitions between no disabilities, IADL disability, BADL disability, and death. At baseline, 299 participants $(50.0 \%)$ were disabled in IADL only, and 155 participants $(26.0 \%)$ were
\end{abstract}

A. H. van Houwelingen · J. Gussekloo • W. P. J. den Elzen • J. W. Blom $(\bowtie)$

Department of Public Health and Primary Care, Leiden University Medical Center, Postzone V0-P, P.O. Box 9600, 2300 RC Leiden, The Netherlands

e-mail: J.W.Blom@lumc.nl

I. D. Cameron

Rehabilitation Studies Unit, Sydney Medical

School-Northern, University of Sydney, Ryde, New South

Wales, Australia

H. Putter

Department of Medical Statistics and BioInformatics, Leiden University Medical Center, Leiden, The Netherlands disabled in both BADL and IADL. During 5-year follow-up, 374 participants $(62.6 \%)$ made $>1$ transition between disability states, mostly deterioration in disability. Males had a lower risk of deterioration [hazard ratio (HR), 0.75 (95 \% CI, 0.58-0.96)] compared to females. No gender differences were observed for improvement [HR, 0.64 (95\% CI, 0.37-1.11)]. Participants with depressive symptoms were less likely to improve [HR, 0.50 (95\% CI, 0.28-0.87)]. Participants with depressive symptoms [HR, 1.46 (95\% CI, 1.12-1.91)], >1 chronic disease [HR, $1.60(95 \% \mathrm{CI}, 1.27-2.01)]$, and with cognitive impairment [HR, 1.60 (95\% CI, 1.20-2.13)] had the highest risk of deteriorating. Disability is a dynamic process in the oldest old. Deterioration is more common than improvement. Older men are less likely to deteriorate than women. The presence of

\author{
S. Kurrle \\ Division of Rehabilitation and Aged Care, Hornsby \\ Ku-ring-gai Health Service, Hornsby, \\ New South Wales, Australia
}

A. J. M. de Craen · A. B. Maier

Department of Gerontology and Geriatrics, Leiden

University Medical Center, Leiden, The Netherlands
A. B. Maier
Section of Gerontology and Geriatrics, Department of Internal Medicine, VU University Medical Center, Amsterdam, The Netherlands 
depressive symptoms, chronic disease, and cognitive impairment predicts deterioration.

Keywords Elderly/aged - Disability in activities of daily living $\cdot$ Recovery of function - Transitions between disability states

$\begin{array}{ll}\text { Abbreviations } \\ \text { IADL } & \text { Instrumental activities of daily living } \\ \text { BADL } & \text { Basic activities of daily living } \\ \text { COPD } & \text { Chronic obstructive pulmonary disease } \\ \text { ATC } & \text { Anatomic Therapeutic Chemical } \\ \text { MMSE } & \text { Mental state examination } \\ \text { GDS-15 } & \text { 15-Item Geriatric Depression Scale } \\ \text { GARS } & \text { Groningen Activities Restriction Scale } \\ \text { PASW } & \text { Predictive Analytics Software } \\ \text { HR } & \text { Hazard ratio }\end{array}$

\section{Introduction}

Disability in older people is associated with dependency, morbidity, and shorter life expectancy (Cigolle et al. 2007; Manton et al. 2008). As the population in the Western world is ageing (Christensen et al. 2009; Lutz et al. 2008), the number of older people facing disability will increase in the coming decades. This increasing prevalence of disability will be accompanied by rising healthcare costs, since care for disabled persons is more costly than care for non-disabled persons (Guralnik et al. 2002; Harrow et al. 1995). Therefore, disability becomes increasingly important for older people, their caregivers, and policymakers.

New preventive strategies directed at disability could diminish the burden of disability. When considering the introduction of new strategies, it is important to know which subgroup of older people is candidates for prevention of disability. Previous research has shown that sex (Nikolova et al. 2011), level of education (Boyd et al. 2009), cognitive decline (Tas et al. 2007; Drewes et al. 2011), physical frailty (Hardy et al. 2005a; b), chronic disease (Stuck et al. 1999; Drewes et al. 2011), prior disability history (Hardy et al. 2006), and depressive symptoms (Stuck et al. 1999; Barry et al. 2009) contribute to changes in disability.

Several studies (Nikolova et al. 2011; Peres et al. 2005; Gill et al. 2010; Hardy and Gill 2004; Hardy et al. 2005; b) including the Precipitating Events
Project (Gill et al. 2001) found that disability is not a static state, but has a dynamic nature, which means that people can move in and out of disability, with continuous transitions between states of disability. However, most studies focused on transitions in disability in basic activities of daily living (BADL) in older individuals without disability. Less attention has been paid to transitions between disability in BADL and disability in instrumental activities of daily living (IADL) in older people in the general population (Nikolova et al. 2011; Peres et al. 2005). Moreover, most studies tended to focus on younger elderly, so that data on disability in individuals aged $\geq 85$ years are scarce (Vaupel 2010).

This study investigates the transitions between no disability in BADL and IADL, and IADL disability, BADL disability, and death, and determines predictors of these transitions in disability states in the oldest old in the general population.

\section{Methods}

Study population

This study was conducted within the Leiden 85-plus study, an observational population-based prospective cohort study of 85-year-old inhabitants of Leiden, the Netherlands. Between 1997 and 1999, 705 inhabitants of Leiden reached the age of 85 years and were eligible for participation. Of these, 14 died before enrolment and 92 refused to participate, resulting in a study population of 599 participants (response rate, $87 \%$ ). No selection criteria for health and demographic characteristics were applied (Bootsma-van der Wiel et al. 2002b).

Participants were followed for 5 years until the age of 90 years or until death. At baseline and annually thereafter, all participants were visited at their place of residence, where face-to-face interviews were conducted, cognitive testing was performed, and information on income, education, and disabilities in BADL and IADL was obtained. For the present study, participants with missing BADL and IADL measurements at baseline were excluded $(n=2)$ resulting in a study population of 597 participants. All participants gave informed consent; for participants with severe cognitive impairment, informed consent was obtained from a proxy. The Medical Ethics Committee of Leiden University Medical Centre approved the study. 
Study parameters

\section{Predictors}

Sociodemographic factors During baseline interviews, a research nurse collected information on sex, residency, income, and level of education.

Presence of chronic diseases Information on participants' medical history was obtained by standardized interviews with their treating general practitioner or elderly care physician, and/or pharmacy records. We obtained clinical information on the presence of diabetes mellitus, chronic obstructive pulmonary disease (COPD), Parkinson's disease, history of stroke and myocardial infarction, cancer, arthritis, and hip fracture. COPD was considered present when the diagnosis COPD was reported by the treating physician or when lung medication [Anatomic Therapeutic Chemical (ATC) code R03] was used at age 85 years. The presence of diabetes mellitus was based on the diagnosis of the treating physician, a non-fasting glucose level $>200 \mathrm{mg} / \mathrm{dL}$, or the use of anti-diabetic medication.

Cognitive function Cognitive function was measured with the Mini-Mental State Examination (MMSE) with scores ranging from 0 to 30 (Heeren et al. 1990). Cognitive impairment was considered present when the MMSE score was below 24 points.

Depressive symptoms The 15-Item Geriatric Depression Scale (GDS-15) was used as a screening instrument for depression (Sheikh and Yesavage 1986). As the validity and the reliability of the GDS-15 may be reduced in participants with severe cognitive impairment, this questionnaire was restricted to those with MMSE scores above 18 points. Depressive symptoms were considered present when the GDS-15 score was 4 points or more. This cut-off was used because of its high sensitivity and its acceptable specificity for depressive symptoms, which has been found in a previous study using the same study setting (De Craen et al. 2003).

\section{Outcome}

Functional status Disability in BADL and IADL was measured annually with the Groningen Activities Restriction Scale (GARS; Kempen et al. 1996). The GARS is a questionnaire that assesses disabilities in competence in nine BADL items and nine IADL items. The GARS is extensively validated in various study populations and has satisfactory clinimetric properties; the test-retest reliability of the GARS-3 was 0.71 (Kempen et al. 2012).

The BADL items included the following: walk inside the house, get up and out of bed, get in and out of a chair, visit the toilet, wash hands and face, wash body, dress and undress, eat and drink, and prepare breakfast (Bootsmavan der Wiel et al. 2001). The IADL items included the following: light housework, heavy cleaning, wash and iron clothes, clean and make the bed, prepare a hot meal, climbing/descending stairs, get around outdoors, do the groceries, and attend to feet and toenails (Bootsma-van der Wiel et al. 2001). Questions were phrased "Can you fully independently...?" We dichotomized the answers into a score of 0 (cannot or only with help from others) or 1 (yes fully independently, with or without difficulty). Disability in BADL or IADL was considered present when a participant responded "Cannot or only with help from others" on any BADL or IADL item, respectively. The dichotomizing of BADL and IADL was done because no universal cutoff points of the GARS are known.

Mortality Dates of death were obtained from municipal registries.

Statistical analysis

\section{Descriptive statistics}

Baseline characteristics and the prevalence of IADL and BADL disability at baseline and during follow-up are presented as number (percentage).

\section{Multi-state model}

Multi-state analysis was used to assess the participants' risk for making transitions between states of disability. Our multi-state representation of disability included four states: (1) no disability in either BADL or IADL, (2) disability in IADL, (3) disability in both BADL and IADL, and (4) death (Fig. 1). Death was included as a competing risk in this model. Since the transition between BADL disability and no disability was observed very rarely, we did not include this transition in the multi-state model.

Transition hazards were first estimated without the inclusion of covariates, then also with the inclusion of 


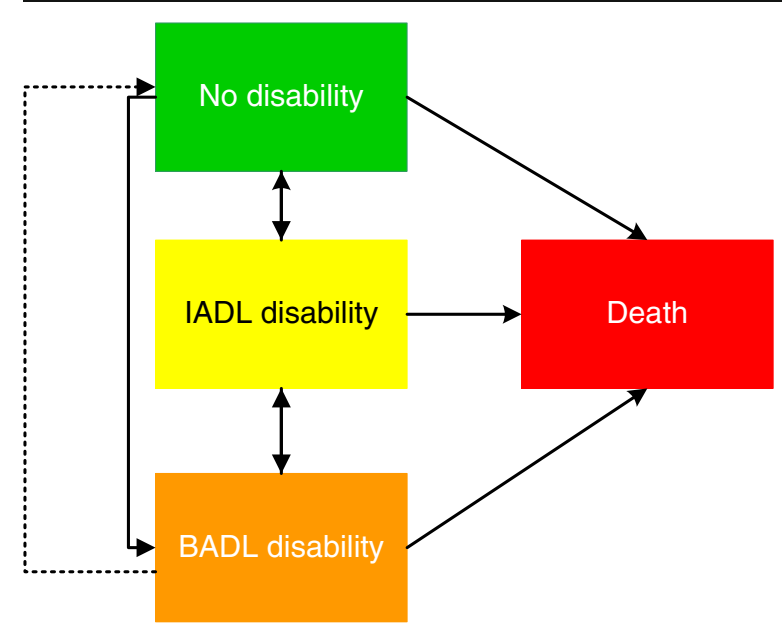

Fig. 1 Multi-state representation of the stages of disability. The boxes represent the four stages. The solid arrows represent the observed transitions; the dotted arrow represents a possible transition that was very rare in this study population and was therefore excluded from the multi-state model

covariates. The Aalen-Johansen estimator (Aalen and Johansen 1978) was used to obtain state occupation probabilities from the transition hazards. The baseline transition hazards of all transitions going into the "death" state were assumed to be proportional (Putter et al. 2007), thus allowing the estimation of hazard ratios of IADL and BADL disability on mortality, compared to having no disability. Estimating covariate effects for all transitions separately would yield too unstable estimates because of rare events for some transitions. Therefore, we hypothesized that the effect of a covariate would be similar in the transition from no disability to IADL disability and from IADL disability to BADL disability, since both these transitions represent deterioration in disability state. Similarly, we assumed that the effect of covariates would be similar in the transition from IADL disability to no disability and from BADL disability to IADL disability, since these transitions represent an improvement in disability state. Moreover, the effect of covariates was also assumed to be the same for transitions from no disability to death, and from IADL disability or BADL disability to death. Therefore, we used a simplified model with deteriorating transitions, improving transitions, and transitions to death. Preliminary analyses showed that this simplification did not result in a model with worse fit.

To study the effect of sex, living situation, income, educational level, presence of chronic disease, cognitive impairment, and depressive symptoms on transitions between disability states, we used these predictors as covariates in the simplified model. Sex, income, education level, living alone, and presence of chronic diseases were included as fixed variables. In the model, cognitive function and depressive symptoms were included as timedependent variables (i.e., each yearly measurement was included in the model).

Descriptive statistics were computed using PASW 17.0 for Windows. The package mstate (de Wreede et al. 2010) version 0.2.5, for $R$ version 2.12.0 (R Development Core Team 2006), was used for the multi-state analysis. A $p$ value of $<0.05$ was considered statistically significant.

\section{Results}

Study population

Table 1 presents the baseline characteristics of the study population at age 85 years. One third of the population was male, and $18 \%$ was living in a longterm care facility. Of all participants, $118(23.6 \%)$ had depressive symptoms, $183(30.7 \%)$ had cognitive impairment (of which 58 had a clinical diagnosis of dementia by their GP), and almost $70 \%$ had at least one chronic disease. At the end of follow-up, 277 participants aged 90 years were interviewed. During the 5-year follow-up period, 41 participants refused further participation, and 279 participants died.

Prevalence of disability

At age 85 years, 143 participants $(24 \%)$ had no disability in IADL and BADL, 299 participants (50 \%) had IADL disability only, and 155 participants (26\%) had BADL disability. Participants with BADL and IADL disability most often reported being unable to do heavy household activities compared with their counterparts with IADL disability only. Being unable to wash and dry the whole body was the most frequently reported BADL item for this group (Web Appendix Table 3). The prevalence of BADL disability increased with age to $58 \%$ at age 90 years. The prevalence of IADL disability and the prevalence of no disability decreased during follow-up to $35 \%$ and $6 \%$, respectively, at age 90 years (Fig. 2). As expected, at each year, all participants with BADL disability also had IADL disability, showing the hierarchical relationship between the two forms of disability. 
Table 1 Baseline characteristics of the participants at age 85 years

$n=597$

\begin{tabular}{ll} 
Sociodemographic & \\
Men & $202(33.8)$ \\
Institutionalized & $107(17.9)$ \\
Low income (<750 euro monthly) & $295(49.3)$ \\
Low education level (6 years of primary school) & $207(34.6)$ \\
General health & \\
Depressive symptoms (GDS-15 $\geq 4$ pts) & $118(23.6)$ \\
Cognitive impairment (MMSE $<24$ pts) & $183(30.7)$ \\
Chronic diseases & \\
$\geq 1$ chronic disease & $414(69.1)$ \\
Cancer & $104(17.4)$ \\
Myocardial infarction & $63(10.6)$ \\
Stroke & $61(10.2)$ \\
Diabetes & $86(14.4)$ \\
Chronic obstructive pulmonary disease & $70(11.7)$ \\
Parkinson's disease & $15(2.5)$ \\
Arthritis & $193(32.3)$ \\
History of hip fracture & $38(6.4)$ \\
\hline
\end{tabular}

Data are numbers and percentages. Chronic diseases included cancer, myocardial infarction, stroke, diabetes, chronic obstructive pulmonary disease, Parkinson's disease, arthritis, and history of hip fracture

GDS-15 Geriatric Depression Scale 15 items, MMSE MiniMental State Examination

Disability states during follow-up

Of the 277 participants $(46.4 \%)$ who were alive at 5-year follow-up, 205 (41.2\%) made one or more transitions between disability states, and $72(12.0 \%)$ were stable in their baseline disability state. Of these 72 participants, five $(0.8 \%)$ remained free of disability during follow-up, $37(6.2 \%)$ had stable IADL disability, and $30(5.0 \%)$ had stable BADL disability. Of the 277 participants who were alive at 5-year follow-up, 83 (30\%) had no disability at baseline, 150 (54.2\%) had IADL disability, and 44 (15.9\%) had BADL disability at baseline.

Of the 279 participants who died during follow-up $(53.4 \%), 110(18.4 \%)$ were stable in their baseline disability state, and 169 participants $(28.3 \%)$ had made one or more transitions between disability states before death.

In total, 374 participants $(69.5 \%)$ made one or more transitions between disability states, and 182 (30.5\%) were stable in their baseline disability state until death or until they reached the age of 90 years. The 374 participants who made one or more transitions between disability states made 579 transitions in total (i.e., mean 1.6 transitions per participant); most of these transitions were directed at deteriorating disability (513 deteriorating transitions and 66 improving transitions). Of these 374 participants, 83 (13.9\%) improved in disability state at some time during follow-up, 29 (4.9\%) improved from IADL disability to no disability state, and 54 participants (9.0\%) improved from BADL disability to IADL disability. The remaining 291 participants made transitions directed at greater disability only.

\section{Transitions between states}

The relative risk for participants with IADL disability to deteriorate to BADL disability was HR 6.21 (95\% CI, 3.35-11.35), compared to participants with no disability to deteriorate to BADL disability. Moreover, an increase in disability was associated with an increased risk of death. The relative mortality risk for participants with IADL disability was HR 1.44 (95\% CI, 0.97-2.63) and for participants with BADL disability was HR 4.20 (95\% CI, 2.31-7.53), both compared to participants with no disability at any time during follow-up.

\section{Predictors of transitions}

Table 2 shows the predictive value of sociodemographic and health determinants on disability transitions. Males had a higher death rate compared to females, but were more stable with respect to their disability state; they had a lower risk of deteriorating [HR, 0.75 (95\% CI, 0.58$0.96), p=0.03]$. No gender differences were observed for improving [HR, $0.64(0.37-1.11), p=0.11]$ in disability state. Higher income (more than state pension), higher education level (more than primary school), and living alone were not associated with improvement or deterioration in disability state. Participants with depressive symptoms (GDS-15 score $>4$ ) had a lower risk of transition to an improved disability state [HR, 0.50 (95\% CI, $0.28-0.87), p=0.01]$, and a higher risk of transition to a deteriorated disability state [HR, 1.46 (1.12-1.91), $p<0.01)$ compared to participants without depressive symptoms. Participants with and without depressive symptoms had similar mortality rates. Chronic disease and cognitive impairment (MMSE score <24) were associated with a higher risk of deteriorating in disability state [HR, 1.60 (95 \% CI, 1.27-2.01) and HR, 1.60 (95\% CI, 
Fig. 2 Estimated transition probabilities of the three states of disability and death during follow-up depending on the disability state at baseline (age 85): a no disability at baseline, b IADL disability at baseline, and $\mathbf{c}$ BADL disability at baseline

\section{a}

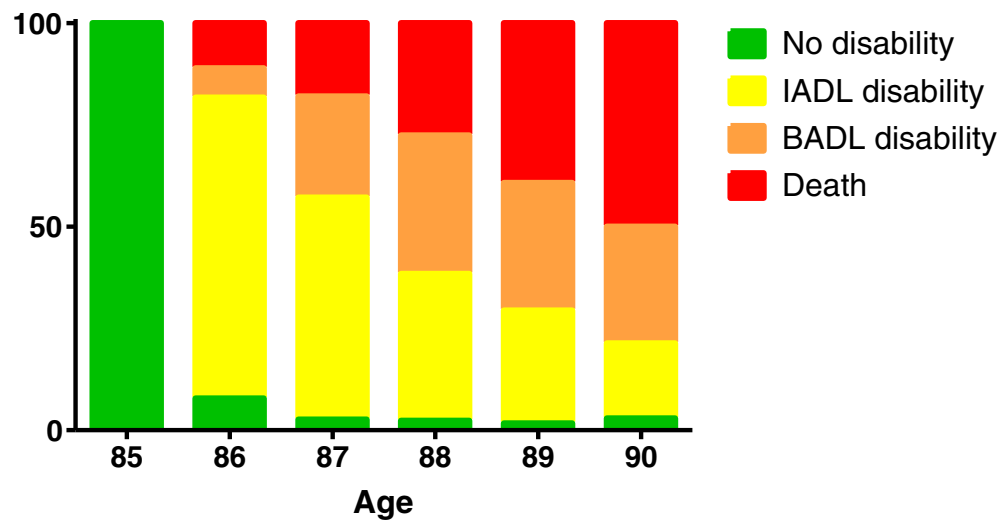

\section{b}

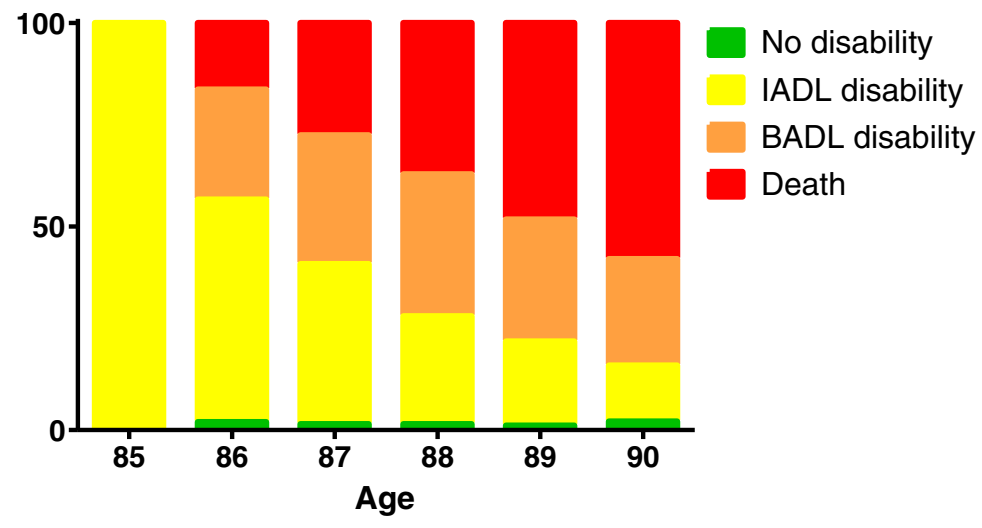

C

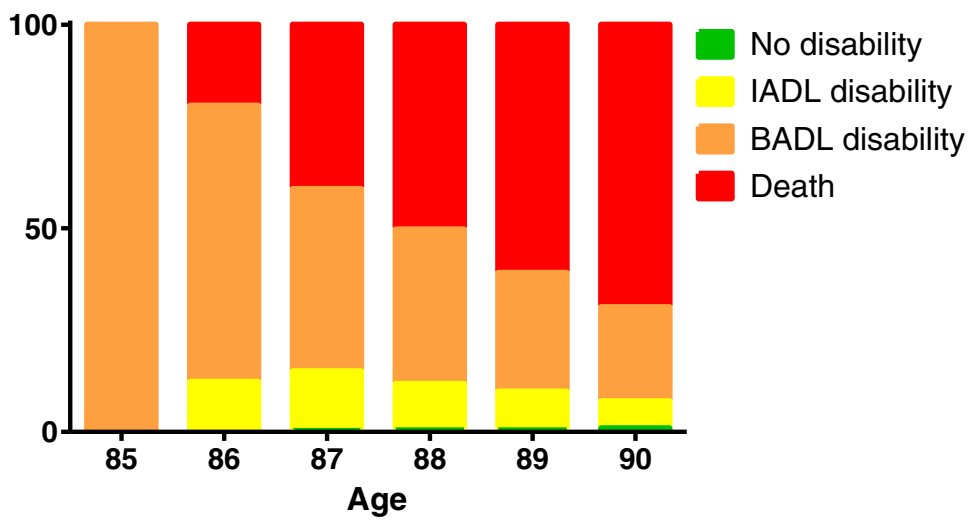

1.20-2.13), respectively, $p<0.01]$ and a higher death rate [HR, 1.48 (95\% CI, 0.96-2.26), $p=0.07$ and HR, 1.64 (1.12-2.39), $p=0.01$ ), but were not associated with an increased or decreased risk for improvement in disability state.

\section{Discussion}

The present study shows that, among the oldest old in the general population, disability in BADL and IADL is a dynamic process with frequent transitions between 
Table 2 Multivariate prediction of improvement in disability state, deterioration in disability state, and death by health characteristics over the 5-year follow-up

\begin{tabular}{|c|c|c|c|c|c|c|}
\hline & \multicolumn{2}{|c|}{$\begin{array}{l}\text { Relative risk of } \\
\text { transition to improved } \\
\text { disability state }\end{array}$} & \multicolumn{2}{|c|}{$\begin{array}{l}\text { Relative risk of } \\
\text { transition to deteriorated } \\
\text { disability state }\end{array}$} & \multicolumn{2}{|l|}{$\begin{array}{l}\text { Relative risk of } \\
\text { transition to death }\end{array}$} \\
\hline & $\mathrm{HR}(95 \% \mathrm{CI})$ & $p$ & $\operatorname{HR}(95 \% \mathrm{CI})$ & $p$ & $\mathrm{HR}(95 \% \mathrm{CI})$ & $p$ \\
\hline Men (ref: women) & $0.64(0.37-1.11)$ & 0.11 & $0.75(0.58-0.96)$ & 0.03 & $2.10(1.44-3.07)$ & $<0.01$ \\
\hline $\begin{array}{l}\text { Low income ( }<750 \text { euro monthly) } \\
\text { (ref: }>750 \text { euro monthly) }\end{array}$ & $1.07(0.67-1.71)$ & 0.77 & $1.10(0.87-1.39)$ & 0.41 & $0.97(0.67-1.41)$ & 0.88 \\
\hline $\begin{array}{l}\text { Low education level (6 years of primary } \\
\text { school) (ref: > primary school) }\end{array}$ & $1.21(0.74-1.98)$ & 0.45 & $0.97(0.77-1.23)$ & 0.82 & $0.94(0.64-1.38)$ & 0.76 \\
\hline Living alone (ref: living with someone) & $1.48(0.92-2.39)$ & 0.11 & $1.13(0.88-1.45)$ & 0.32 & $0.94(0.64-1.38)$ & 0.73 \\
\hline Chronic disease (ref: no chronic disease) & $0.76(0.47-1.24)$ & 0.27 & $1.60(1.27-2.01)$ & $<0.01$ & $1.48(0.96-2.26)$ & 0.07 \\
\hline Cognitive impairment (MMSE $<24$ pts) (ref: MMSE $\geq 24)$ & $0.62(0.36-1.09)$ & 0.10 & $1.60(1.20-2.13)$ & $<0.01$ & $1.64(1.12-2.39)$ & 0.01 \\
\hline Depressive symptoms (GDS-15 $\geq 4$ pts) (ref: GDS $<4$ ) & $0.50(0.28-0.87)$ & 0.01 & $1.46(1.12-1.91)$ & $<0.01$ & $1.09(0.75-1.58)$ & 0.64 \\
\hline
\end{tabular}

Hazard ratios were estimated with a multistate Cox model. Sex, income, education level, living alone, and presence of chronic diseases were included as fixed variables. Cognitive function and depressive symptoms were included as time-dependent variables. Chronic diseases included cancer, myocardial infarction, stroke, diabetes, chronic obstructive pulmonary disease, Parkinson's disease, and history of hip fracture

$C I$ confidence interval, GDS-15 Geriatric Depression Scale 15 items, MMSE Mini-Mental State Examination, HR hazard ratio

disability states. In this study, the most important predictors for transition from no disability to both IADL and BADL disability states were the presence of depressive symptoms, chronic disease, and cognitive impairment. Men had a higher mortality rate compared to women, but were more stable with respect to their disability state. Whilst transitions to greater disability and death were much more common than improvements in disability, a small number of very old persons do improve in disability status. Recovery from any acute pathological condition or injury could partly explain this. An intriguing result of this study is that the proportion of older people without any disability that shows some functional decline between age 85 and age 86 is almost $80 \%$ (similarly from age 86 to age 87). This may be explained by the fact that people of this advanced age are known to decline in function very rapidly (McCusker et al. 2002).

Our results in persons aged $\geq 85$ years confirm those of other studies on transitions between disability states (Nikolova et al. 2011; Gill et al. 2010; Hardy and Gill 2004; Hardy et al. 2005; b; Peres et al. 2005; Mor et al. 1994). However, unlike other studies which presented high recovery rates (Boyd et al. 2009; Hardy and Gill 2004; Hardy et al. 2005; b; Peres et al. 2005; Nikolova et al. 2011), our study participants infrequently recovered from disability. Three of these previous studies were performed in the same study population (Hardy and Gill 2004; Hardy et al. 2005; b) of the Precipitating Events Project (Gill et al.
2001). Of the other three, one was restricted to moderately and severely disabled women (Boyd et al. 2009), and one to disabled patients aged 65 years and older registered for home services (Nikolova et al. 2011). Only the populationbased study by Peres et al. and the study of Mor et al. are comparable with the present study (Peres et al. 2005; Mor et al. 1994). In these studies, similar results were found for people of old age (80 years and over). The infrequent recovery in our study might thus be explained by the old age of our study population.

Moreover, since IADL disability is highly prevalent among the very elderly (Bootsma-van der Wiel et al. 2001), it is less likely that the oldest old fully recover to a non-disabled state. Therefore, the transition from BADL disability to no disability was too uncommon to include in the multistate model. In contrast to the abovementioned studies, we had yearly intervals between disability assessments.

Our predictors of transitions in disability state are generally in line with others, who also found that males are more stable with respect to their disability state than females. This has been described as the health-survival paradox in the very old: i.e., men seem to be healthier than women but die earlier (Oksuzyan et al. 2008). The biological mechanism underlying this paradox has yet to be unraveled.

The fact that both cognitive impairment and the presence of chronic illness predicted a greater risk of 
deterioration in disability confirms the results of previous studies on this topic (Bootsma-van der Wiel et al. 2002a; Drewes et al. 2011; Nikolova et al. 2011; Peres et al. 2005; Stuck et al. 1999; Tas et al. 2007). Older people with depressive symptoms were at higher risk of deteriorating in disability state, and had a lower chance of improvement. This is consistent with others (Wang et al. 2002; Barry et al. 2009) and provides evidence that recognition and treatment of depressive symptoms in the oldest old could lead to a reduction of the burden of disability.

The aim of our study was to investigate predictors of disability status in the general population at large. Our study population therefore also included persons living in care homes or nursing homes. Since persons living in these institutions are not likely to improve in disability status, and may often have an increased risk of depression (Boorsma et al. 2012), comorbidity, and cognitive impairment, this may have resulted in an underestimation of the predictive value of depression, comorbidity, and cognitive impairment on the change in disability status for community-dwelling elderly.

Since this study assesses the predictive value of the determinants on disability changes, the results should not be etiologically interpreted. For example, the predictive value of depression on disability has been extensively studied, but a causal relationship has not been identified yet (Barry et al. 2013).

The results of our study are important for several reasons. First, people aged $\geq 85$ years are the fastest growing segment of the general population, and data on disability in this population are limited (Vaupel 2010). This age group is often excluded from clinical trials and preventive interventions, and is sometimes undertreated (Habicht et al. 2008; de Boer et al. 2010; Fracheboud et al. 2006). Therefore, the results of this study in this specific population provide valuable information to help develop preventive strategies directed towards disability in this age group. Second, the results could also have implications for geriatric rehabilitation programs.

\section{Strengths and limitations}

Our study has several strengths. The Leiden 85-plus study is a longitudinal population-based cohort study with no exclusion criteria and almost complete follow-up for mortality. A total of $87 \%$ of the oldest old in the population participated in this study. This high participation rate adds to the external validity of our results. Moreover, the longitudinal design with repeated measurements of competence in IADL and BADL allowed us to demonstrate the dynamic process of disability in this unique sample of very old participants. Another strength of our study is that we included both IADL and BADL disability and the transitions between these disability states, healthy, and death. In addition, the use of a multi-state model allowed us to include more than one transition per participant, and allowed inclusion of death as a competing risk and separate outcome.

A limitation of our study is its relatively small sample size. A larger study would allow us to investigate the predictive effect of more covariates and to perform subgroup analyses. Another limitation is that, due to the dichotomizing of disability in this study, small changes in disability status may be unnoticed by the multi-state model. This relates to the limits of the multi-state model, which does not allow inclusion of continuous variables. Moreover, since the multistate model will be unstable when too many predictors are added, we added a predictor which was based on a score of all chronic diseases.

\section{Conclusion}

Disability remains a dynamic process in the oldest old in the general population. Transitions to a deteriorating disability status are more common than transitions to an improved disability status. Older men are less likely to deteriorate in disability status than women. The presence of depressive symptoms, chronic disease, and cognitive impairment predict deterioration in disability. Further research is needed to investigate whether older people with a higher risk for deterioration would benefit from preventive programs or interventions designed to reverse disability.

\section{Acknowledgments}

Authors' contributions Professor Gussekloo had full access to all data in the study, and takes responsibility for the integrity of the data and the accuracy of the data analysis. Study concept and design was done by J. Gussekloo. Acquisition of data was performed by A.J.M. de Craen and J. Gussekloo. Analyses and interpretation of data were performed by A.H. van Houwelingen, I.D. Cameron, J. Gussekloo, H. Putter, S. Kurrle, W.P.J. den Elzen, A.J.M. de Craen, A.B.. Maier, and J.W. Blom. Drafting of the manuscript was done by A.H. van Houwelingen, W.P.J. den Elzen, and J.W. Blom. Critical revision of the manuscript for important intellectual content was performed by A.H. van Houwelingen, I.D. Cameron, J. Gussekloo, H. Putter, S. Kurrle, W.P.J. den Elzen, A.J.M. de Craen, A.B.. Maier, and J.W. Blom. Statistical analysis was done by A.H. van Houwelingen and $\mathrm{H}$. 
Putter. J. Gussekloo obtained funding. W.P.J. den Elzen, A.J.M. de Craen, and J. Gussekloo provided administrative, technical, or material support. Study supervision was performed by J. Gussekloo A grant was also received from the Dutch Organisation of Scientific Research (NWO) for Open Access publication of this manuscript.

Funding The Leiden 85 -plus study was funded (in part) by an unrestricted grant from the Dutch Ministry of Health, Welfare and Sports (1997-2001). The sponsor had no role in the design and conduct of the study; in the collection, analysis, and interpretation of the data; or in the preparation, review, or approval of the manuscript.
Conflicts of interest The authors confirm that there are no conflicts of interest. All authors read and approved the final version of the manuscript.

Open Access This article is distributed under the terms of the Creative Commons Attribution License which permits any use, distribution, and reproduction in any medium, provided the original author(s) and the source are credited.

\section{Appendix}

Table 3 The prevalence of disability on the individual BADL and IADL items of the GARS at age $85(n=597)$

\begin{tabular}{|c|c|c|c|c|}
\hline Item & $\begin{array}{l}\text { Total population } \\
n=597\end{array}$ & $\begin{array}{l}\text { No disability } \\
n=143\end{array}$ & $\begin{array}{l}\text { IADL disability }^{\mathrm{a}} \\
n=299\end{array}$ & $\begin{array}{l}\text { BADL disability } \\
n=155\end{array}$ \\
\hline
\end{tabular}

BADL: unable to

\begin{tabular}{|c|c|c|c|c|}
\hline (1) Dress yourself & $103(17.3)$ & 0 & 0 & $103(66.5)$ \\
\hline (2) Get in and out of bed & $53(8.9)$ & 0 & 0 & $53(34.2)$ \\
\hline (3) Stand up from sitting in a chair & $53(8.9)$ & 0 & 0 & $53(54.2)$ \\
\hline (4) Wash face and hands & $45(7.5)$ & 0 & 0 & $45(29.0)$ \\
\hline (5) Wash and dry whole body & $124(20.8)$ & 0 & 0 & $124(80.0)$ \\
\hline (6) Go to the toilet & $60(10.1)$ & 0 & 0 & $60(38.7)$ \\
\hline (7) Eat and drink & $21(3.5)$ & 0 & 0 & $21(13.5)$ \\
\hline (8) Get around in the house & $67(11.2)$ & 0 & 0 & $67(43.2)$ \\
\hline (12) Prepare breakfast & $64(10.7)$ & 0 & 0 & $64(41.3)$ \\
\hline \multicolumn{5}{|l|}{ ADL: unable to } \\
\hline (9) Go up and down the stairs & $225(37.7)$ & 0 & $89(29.8)$ & $136(87.7)$ \\
\hline (10) Walk outdoors & $171(28.6)$ & 0 & $50(16.7)$ & $121(78.1)$ \\
\hline (11) Take care of your feet and toenails & $340(57.0)$ & 0 & $195(65.2)$ & $145(93.5)$ \\
\hline (13) Prepare dinner & $209(35.0)$ & 0 & $80(26.8)$ & $129(83.2)$ \\
\hline (14) Light household activities & $127(21.3)$ & 0 & $28(9.4)$ & $99(63.9)$ \\
\hline (15) Heavy household activities & $350(58.6)$ & 0 & $200(66.9)$ & $150(96.8)$ \\
\hline (16) Wash and iron clothes & $223(37.4)$ & 0 & $94(31.4)$ & $129(83.2)$ \\
\hline (17) Make the beds & $207(34.7)$ & 0 & $80(26.8)$ & $127(81.9)$ \\
\hline (18) Groceries & $231(38.7)$ & 0 & $105(35.1)$ & $126(81.3)$ \\
\hline
\end{tabular}

Data represent numbers (percent)

a Disability in BADL or IADL was considered present when a participant responded "Cannot or only with help from others" on any BADL or IADL item, respectively

\section{References}

Aalen OO, Johansen S (1978) Empirical transition matrix for nonhomogeneous Markov-chains based on censored observations. Scand J Stat 5:141-150

Barry LC, Allore HG, Bruce ML, Gill TM (2009) Longitudinal association between depressive symptoms and disability burden among older persons. J Gerontol A Biol Sci Med Sci 64:1325-1332
Barry LC, Soulos PR, Murphy TE, Kasl SV, Gill TM (2013) Association between indicators of disability burden and subsequent depression among older persons. J Gerontol A Biol Sci Med Sci 68:286-292

Boorsma M, Joling K, Dussel M, Ribbe M, Frijters D, van Marwijk H, Nijpels G, van Hout H (2012) The incidence of depression and its risk factors in Dutch nursing homes and residential care homes. Am J Geriatr Psychiatr: Off J Am Assoc Geriatr Psychiatr 20:932-942 
Bootsma-van der Wiel A, Gussekloo J, De Craen AJ, Van Exel E, Knook DL, Lagaay AM, Westendorp RG (2001) Disability in the oldest old: "can do" or "do do"? J Am Geriatr Soc 49:909 914

Bootsma-van der Wiel A, Gussekloo J, De Craen AJ, Van Exel E, Bloem BR, Westendorp RG (2002a) Common chronic diseases and general impairments as determinants of walking disability in the oldest-old population. J Am Geriatr Soc 50:1405-1410

Bootsma-van der Wiel A, Van Exel E, De Craen AJ, Gussekloo J, Lagaay AM, Knook DL, Westendorp RG (2002b) A high response is not essential to prevent selection bias: results from the Leiden 85-plus study. J Clin Epidemiol 55:1119-1125

Boyd CM, Ricks M, Fried LP, Guralnik JM, Xue QL, Xia J, Bandeen-Roche K (2009) Functional decline and recovery of activities of daily living in hospitalized, disabled older women: the Women's Health and Aging Study I. J Am Geriatr Soc 57:1757-1766

Christensen K, Doblhammer G, Rau R, Vaupel JW (2009) Ageing populations: the challenges ahead. Lancet 374:1196-1208

Cigolle CT, Langa KM, Kabeto MU, Tian Z, Blaum CS (2007) Geriatric conditions and disability: the Health and Retirement Study. Ann Intern Med 147:156-164

de Boer MJ, Ottervanger JP, Suryapranata H, Hoorntje JC, Dambrink JH, Gosselink AT, van't Hof AW, Zijlstra F (2010) Old age and outcome after primary angioplasty for acute myocardial infarction. J Am Geriatr Soc 58:867-872

De Craen AJ, Heeren TJ, Gussekloo J (2003) Accuracy of the 15item geriatric depression scale (GDS-15) in a community sample of the oldest old. Int J Geriatr Psychiatr 18:63-66

de Wreede LC, Fiocco M, Putter H (2010) The mstate package for estimation and prediction in non- and semi-parametric multi-state and competing risks models. Comput Methods Programs Biomed 99:261-274

Development Core Team R (2006) A language and environment for statistical computing. R Development Core Team, Vienna

Drewes YM, den Elzen WP, Mooijaart SP, De Craen AJ, Assendelft WJ, Gussekloo J (2011) The effect of cognitive impairment on the predictive value of multimorbidity for the increase in disability in the oldest old: the Leiden 85plus study. Age Ageing 40:352-357

Fracheboud J, Groenewoud JH, Boer R, Draisma G, de Bruijn AE, Verbeek AL, de Koning HJ (2006) Seventy-five years is an appropriate upper age limit for population-based mammography screening. Int J Cancer 118:2020-2025

Gill TM, Desai MM, Gahbauer EA, Holford TR, Williams CS (2001) Restricted activity among community-living older persons: incidence, precipitants, and health care utilization. Ann Intern Med 135:313-321

Gill TM, Allore HG, Gahbauer EA, Murphy TE (2010) Change in disability after hospitalization or restricted activity in older persons. JAMA 304:1919-1928

Guralnik JM, Alecxih L, Branch LG, Wiener JM (2002) Medical and long-term care costs when older persons become more dependent. Am J Public Health 92:1244-1245
Habicht DW, Witham MD, McMurdo ME (2008) The underrepresentation of older people in clinical trials: barriers and potential solutions. J Nutr Health Aging 12:194-196

Hardy SE, Gill TM (2004) Recovery from disability among community-dwelling older persons. JAMA 291:1596-1602

Hardy SE, Gill TM (2005) Factors associated with recovery of independence among newly disabled older persons. Arch Intern Med 165:106-112

Hardy SE, Dubin JA, Holford TR, Gill TM (2005) Transitions between states of disability and independence among older persons. Am J Epidemiol 161:575-584

Hardy SE, Allore HG, Guo Z, Dubin JA, Gill TM (2006) The effect of prior disability history on subsequent functional transitions. J Gerontol A Biol Sci Med Sci 61:272-277

Harrow BS, Tennstedt SL, McKinlay JB (1995) How costly is it to care for disabled elders in a community setting? Gerontologist 35:803-813

Heeren TJ, Lagaay AM, von Beek WC, Rooymans HG, Hijmans W (1990) Reference values for the Mini-Mental State Examination (MMSE) in octo- and nonagenarians. J Am Geriatr Soc 38: 1093-1096

Kempen GI, Miedema I, Ormel J, Molenaar W (1996) The assessment of disability with the Groningen Activity Restriction Scale. Conceptual framework and psychometric properties. Soc Sci Med 43:1601-1610

Kempen G, Doeglas D, Suurmeijer T (2012) Groningen Activities Restriction Scale (GARS): een handleiding, 2nd edn. UMCG/ Rijksuniversiteit Groningen, Research Institute SHARE, Groningen

Lutz W, Sanderson W, Scherbov S (2008) The coming acceleration of global population ageing. Nature 451:716-719

Manton KG, Gu X, Lowrimore GR (2008) Cohort changes in active life expectancy in the U.S. elderly population: experience from the 1982-2004 National Long-Term Care Survey. J Gerontol B Psychol Sci Soc Sci 63:S269-S281

McCusker J, Kakuma R, Abrahamowicz M (2002) Predictors of functional decline in hospitalized elderly patients: a systematic review. J Gerontol A Biol Sci Med Sci 57:M569-M577

Mor V, Wilcox V, Rakowski W, Hiris J (1994) Functional transitions among the elderly: patterns, predictors, and related hospital use. Am J Public Health 84:1274-1280

Nikolova R, Demers L, Beland F, Giroux F (2011) Transitions in the functional status of disabled community-living older adults over a 3-year follow-up period. Arch Gerontol Geriatr 52:12-17

Oksuzyan A, Juel K, Vaupel JW, Christensen K (2008) Men: good health and high mortality. Sex differences in health and aging. Aging Clin Exp Res 20:91-102

Peres K, Verret C, Alioum A, Barberger-Gateau P (2005) The disablement process: factors associated with progression of disability and recovery in French elderly people. Disabil Rehabil 27:263-276

Putter H, Fiocco M, Geskus RB (2007) Tutorial in biostatistics: competing risks and multi-state models. Stat Med 26:23892430 
Sheikh J, Yesavage J (1986) Geriatric Depression Scale (GDS): recent evidence and development of a shorter version. In: Brink T (ed) Clinical gerontology: a guide to assessment and intervention. The Haworth Press, New York, pp 165-173

Stuck AE, Walthert JM, Nikolaus T, Bula CJ, Hohmann C, Beck JC (1999) Risk factors for functional status decline in community-living elderly people: a systematic literature review. Soc Sci Med 48:445-469
Tas U, Verhagen AP, Bierma-Zeinstra SM, Odding E, Koes BW (2007) Prognostic factors of disability in older people: a systematic review. Br J Gen Pract 57:319-323

Vaupel JW (2010) Biodemography of human ageing. Nature 464:536-542

Wang L, van Belle G, Kukull WB, Larson EB (2002) Predictors of functional change: a longitudinal study of nondemented people aged 65 and older. J Am Geriatr Soc 50:1525-1534 